\section{Out of joint: new insights into the role of commensal gut bacteria in autoimmune arthritis}

It has long been appreciated that inflammation in the intestine can give rise to inflammation at extraintestinal sites, such as the skin, eyes, and joints. Transient spondyloarthritis occurs in $10-20 \%$ of patients with inflammatory bowel disease (IBD), usually during a flare of intestinal inflammation. ${ }^{1}$ Current theories on the pathogenesis of IBD posit that inflammation results from an inappropriate immune response to gut bacteria in genetically predisposed individuals. Advances in metagenomic analysis of the gut microbiota have revealed that changes in the composition of the gut microbiota are commonly associated with $I B D$, although it remains unclear whether these changes are the cause or the result of intestinal inflammation. Alterations in the gut microbiota have also been reported in patients with early rheumatoid arthritis, ${ }^{2}$ supporting a possible connection between immune responses to gut bacteria and development of joint inflammation. A recent Commentary in Mucosal Immunology ${ }^{3}$ discussed the surprising discovery that a single commensal species-segmented filamentous bacteria (SFB) - can skew the phenotype of mucosal CD4+ helper T cells, specifically enhancing the development of interleukin-17 (IL-17) ${ }^{+}$Thelper type 17 (Th17) cells. ${ }^{4,5}$ The SFB-induced increase in the Th17 cell population may improve protection against infection with enteric pathogens, but it carries the potential risk of inflammatory tissue damage and autoimmunity. New evidence has now emerged that SFB in the gut can drive autoimmune arthritis via Th17 cells.

A study published by Wu et al. in the 25 June 2010 issue of $/$ mmunity ${ }^{6}$ demonstrated that the presence of commensal microbes was required for development of autoimmunity and joint inflammation in the $\mathrm{K} / \mathrm{BxN}$ mouse model of autoimmune arthritis, and that SFB alone was sufficient to trigger this pathology. $\mathrm{K} / \mathrm{BxN}$ mice, which were generated by crossing a T-cell receptor (TCR) transgenic line with the NOD strain, spontaneously develop a joint disease that has many features in common with rheumatoid arthritis in humans. In these mice, major histocompatibility complex class II-restricted presentation of a self-peptide derived from glucose-6-phosphate isomerase (GPI) to T cells displaying the transgenic TCR results in expansion of autoreactive T cells that provide help to GPI-specific $B$ cells, resulting in high titers of anti-GPI autoantibodies. An interesting feature of this model is the generation of anti-GPI Th17 cells in the intestinal lamina propria that migrate to the spleen and promote the development of germinal centers and anti-GPI antibody-secreting cells. Joint inflammation in $\mathrm{K} / \mathrm{BxN}$ mice results from a self-sustaining innate immune response initiated by GPI:antiGPI immune complexes. Wu and colleagues found that $\mathrm{K} / \mathrm{BxN}$ mice maintained under germ-free (GF) conditions had reduced antiGPI autoantibody titers, Th17 cells, and joint inflammation as compared with genetically identical mice with a specific pathogen-free (SPF) gut microbiota. Transfer of GF K/ BxN mice to an SPF facility rapidly induced symptoms of arthritis, which could be prevented by treatment with IL-17-neutralizing antibodies. Significantly, monocolonization of GF K/BxN mice with SFB was sufficient to drive production of autoantibodies and pathogenic Th17 cells as well as to trigger arthritis. These results provide the first evidence that commensal bacteria can drive autoimmune arthritis by inducing a Th17 response in the intestine.

This important discovery offers new insights into the contribution of commensal gut bacteria and Th17 cells to the development of arthritis as a complication of intestinal inflammation. The potential relevance of this mechanism to human disease is supported by the recent report that frequencies of Th17 cells in synovial fluid and levels of IL-17 production were correlated with disease activity in patients with autoimmune arthritis. ${ }^{7}$ Given the importance of IL-23R signaling in Th17 development, it is significant that polymorphisms in the IL-23R gene that have been shown to be protective against IBD were also found to be associated with a reduced risk of ankylosing spondylitis. ${ }^{8}$ A mechanism involving gut bacteria-induced Th17 cells may also explain the recent finding of increased numbers of Th17 cells in the synovial fluid of patients with reactive arthritis, a form of spondyloarthropathy that is initiated by infection with enteric bacterial pathogens. ${ }^{9}$ A better understanding of the association of SFB and other gut bacteria with arthritis may lead to the development of new strategies for the prevention and treatment of extraintestinal complications of IBD and enteric infections.

\section{Charlotte Slayton Kaetzel, Associate Editor}

(C) 2010 Society for Mucosal Immunology

1. De Vos, M. Joint involvement associated with inflammatory bowel disease. Dig. Dis. 27, 511515 (2009).

2. Vaahtovuo, J., Munukka, E., Korkeamaki, M., Luukkainen, R., \& Toivanen, P. Fecal microbiota in early rheumatoid arthritis. J. Rheumatol. $\mathbf{3 5}$, 1500-1505 (2008).

3. Ivanov, I.I. \& Littman, D.R. Segmented filamentous bacteria take the stage. Mucosal Immunol. 3, 209-212 (2010).

4. Ivanov, I.I. et al. Induction of intestinal Th17 cells by segmented filamentous bacteria. Cell 139 485-498 (2009).

5. Gaboriau-Routhiau, V. et al. The key role of segmented filamentous bacteria in the coordinated maturation of gut helper $\mathrm{T}$ cell responses. Immunity 31, 677-689 (2009).

6. Wu, H.-J. et al. Gut-residing segmented filamentous bacteria drive autoimmune arthritis via T helper 17 cells. Immunity $\mathbf{3 2}$, 815-827 (2010).

7. Leipe, J. et al. Th17 cells in autoimmune arthritis. Arthritis. Rheum.; e-pub ahead of print 25 June 2010.

8. Rahman, P. et al. Association of interleukin-23 receptor variants with ankylosing spondylitis. Arthritis. Rheum. 58, 1020-1025 (2008).

9. Shen, H., Goodall, J.C., \& Gaston, J.S Frequency and phenotype of Thelper 17 cells in peripheral blood and synovial fluid of patients with reactive arthritis. J. Rheumatol., e-pub ahead of print 15 July 2010. 